\title{
LUIS CIFUENTES DELATTE (1907-2005). SEMBLANZA BIOGRÁFICA
}

\author{
Mariano Pérez Albacete
}

Servicio de Urología. Hospital Universitario Virgen de la Arrixaca. Murcia. España.

\begin{abstract}
Resumen.- OBJETIVO: Con ocasión de cumplirse el centenario del nacimiento del Dr. D. Luis Cifuentes Delatte, y al considerarlo como una de las personalidades de mayor trascendencia de la Urología española de la segunda mitad del siglo XX, repasamos su vida, su entorno y sus relaciones y resaltamos sus vivencias, además de su obra y su faceta investigadora.
\end{abstract}

MÉTODOS: Lectura de sus libros y artículos varios, de las publicaciones periódicas urológicas españolas desde 1930, de las que entresacamos las referencias que sobre él encontramos y también de nuestra relación y conocimiento personal. 30120 El Palmar. Murcia. (España). Mariano.perez2@carm.es
RESULTADO/CONCLUSIONES: Con una importante preparación clínica e investigadora, una amplia capacidad de trabajo, observador juicioso y agudo, además de objetivo y riguroso en la comprobación de sus resultados, el análisis del sedimento urinario, la infección de orina junto con la formación de los cálculos y el estudio de la patología vesical, neurogénica, tumoral y cistopatías, fueron los temas fundamentales a los que se dedicó a la par que destacó sobremanera en la práctica de la cirugía transuretral. Publicista de valía, destaca por la trascendencia de sus escritos, y especialmente por ser maestro de una amplia generación de urólogos españoles e hispanoamericanos y por su entrega al estudio y a la Urología.

Palabras clave: Historia de la Urología. Luis Cifuentes Delatte. Semblanzas urológicas.

Summary.- OBJECTIVES: With the centenary of the birth of Dr. Luis Cifuentes Delatte, considering he in as one of the most transcendental personalities in the Spanish urology of the second half of the 20th-century, we review his life, his environment, and his relationships, and emphasize his life experiences, in addition to his works and research.

METHODS: We read his books and articles from Spanish urological journals from 1930, from which we selected the references we found about him. We also used as a source our personal relationship with him.

RESULTS/CONCLUSIONS: He had an important clinical and research education, a great working capacity; he was a judicious and acute observer, also objective and rigorous checking results; the analysis of urine sediment, urinary tract infection with stone formation, and the study of bladder, neurogenic and tumoral pathologies, as well as a cystopathies, were the main topics he dedicated to; he also was outstanding in transurethral 
surgery. Valuable publicist, he is outstanding due to the transcendence of his written works, and especially for being the Master of an ample generation of Spanish and South American urologists, and for his love for study and urology.

Keywords: History of urology. Dr. Luis Cifuentes Delatte. Urological life sketches.

Nacido en Madrid, el 12 de noviembre de 1907 (Figura 1), hijo del cirujano urólogo Dr. D. Pedro Cifuentes Díaz, (1880-1960), heredó de su padre la entrega a la especialidad. Estudió Medicina en la Facultad de la Universidad Complutense, fue alumno interno en el Hospital Clínico de San Carlos y en el de la Princesa de Madrid, discípulo del Dr. D. Carlos Jiménez Díaz, a quien siempre mantuvo una extraordinaria admiración y respeto y quien le inculcó su amor por la investigación. Finaliza la licenciatura en 1930 y asiste al Congreso Francés de Urología de ese año. Miembro de la Asociación

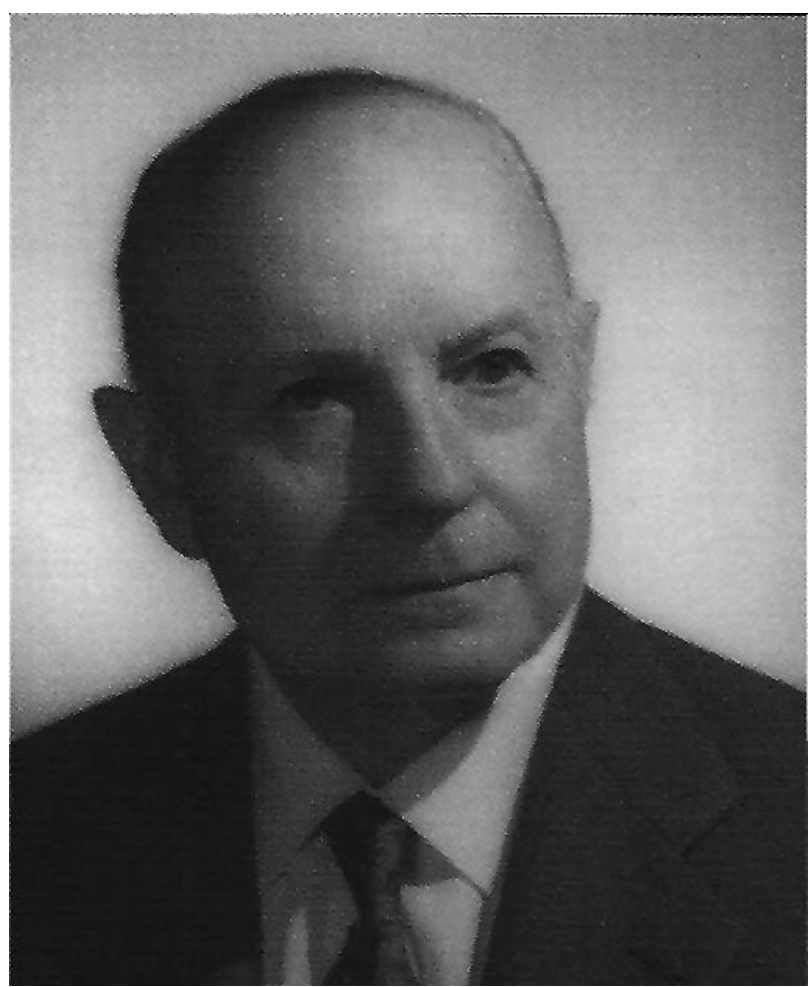

FIGURA 1. Dr. D. Luis Cifuentes Delatte.
Española de Urología, AEU, en 1932, comienza a presentar trabajos clínicos en sus congresos, así en el desarrollado en Coimbra en julio de ese año, el IX de la AEU y III Hispano-Portugués, expone Contribución al estudio de la cistitis (Figura 2), en la que su espíritu inquisidor, ya en esas tempranas fechas, se interesa por el conocimiento de la bacteriología en las afecciones urinarias, cuyo papel desentrañará a lo largo de sus investigaciones sobre la patología de la mucosa vesical y sobre la que insistirá en el $X$ de la AEU y IV Hispano-Portugués, de 1935, en Cádiz, con Los focos extraurinarios en las cistitis. Su primera publicación data de 1934, Obstrucción ureteral doble por intensa piuria.

Realiza su tesis doctoral, Alteraciones del equilibrio ácido base en las nefropatías, leída en 1935 (Figura 3), texto sobre el que ya había presentado una comunicación en el IX Congreso de la $\mathrm{AEU}$, Sobre las alteraciones postoperatorias de la cloremia, con lo que adquiere un amplio conocimiento electrolítico y de la función renal y que fue la base

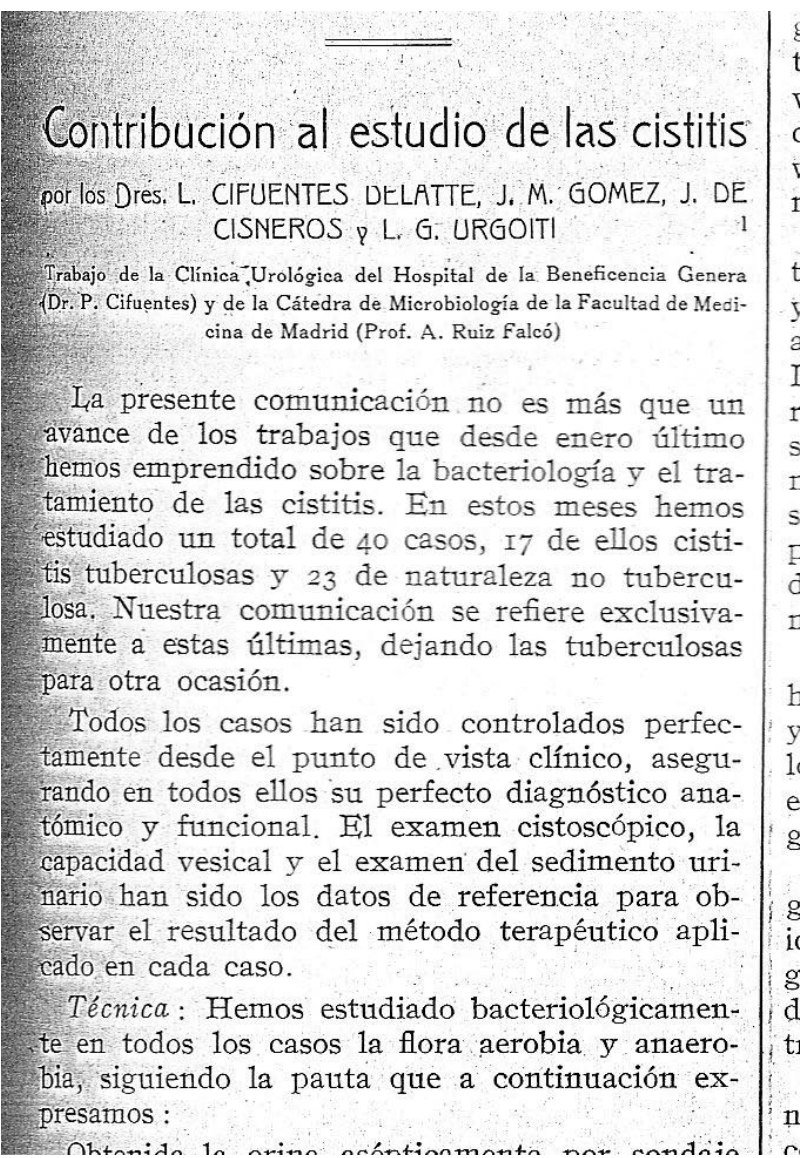

FIGURA 2. Contribución al estudio de las cistitis. La Medicina Íbera, 1932. 
preparatoria de su libro Nefropatías (Enfermedad de Bright), editado en 1943, con el que se adentra en el terreno de la nefrología.

Su preparación científica comenzó con el aprendizaje de idiomas, el francés durante los cursos de bachillerato en el madrileño colegio de El Pilar, cuyo último año lo realizó en un liceo de París, y que más tarde cultivó durante su asistencia al Hospital Necker para ampliar su formación en la especialidad, además de mantener, durante toda su vida, una continúa relación y buena amistad con urólogos franceses y acudir periódicamente a sus congresos.

El alemán lo ejercitó en su época de estudiante tras una estancia en Friburgo, en el Instituto de Fisiología del profesor Hofmann, donde se inició en las técnicas de investigación, $y$, posteriormente, a lo largo de su permanencia en Berlín con el profesor Von Lichtenberg en 1933, para completar su adiestramiento urológico y el inglés lo estudió solo y lo perfeccionó en Estados Unidos en 1946; finalizada la segunda guerra mundial acude para conocer la Urología norteamericana al Hospital de la Universidad de Michigan, donde el Dr. Nesbit le enseña la cirugía endoscópica que prosigue en Chicago con los Drs. Flokcs y Alcocks, además de pasar por la Clínica Mayo, en Rochester.

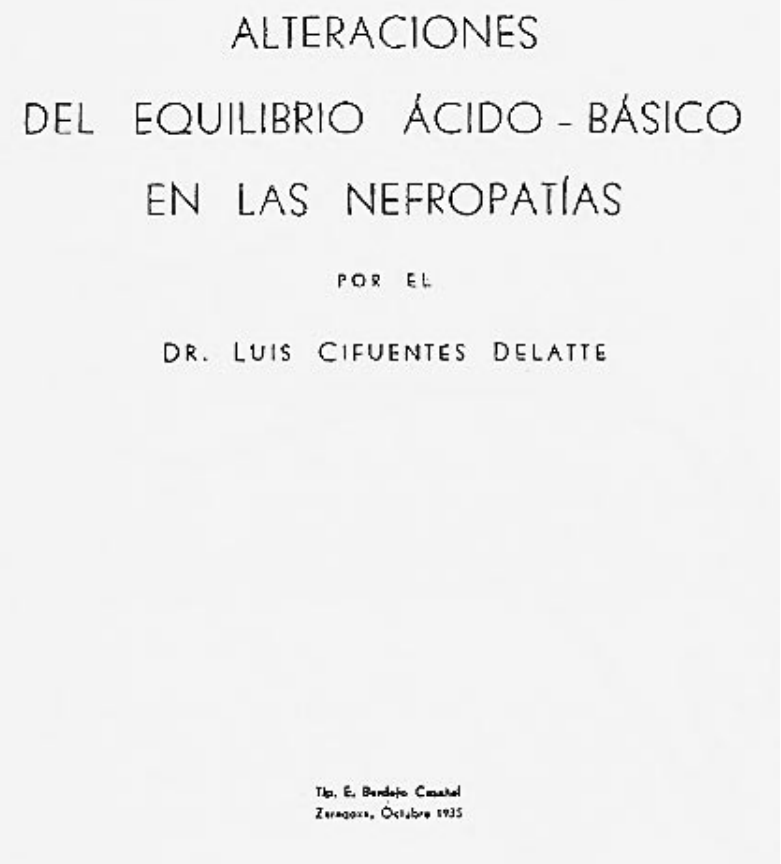

FIGURA 3. Tesis doctoral Alteraciones del equilibrio ácido base en las nefropatías, Zaragoza, 1935.
Durante la Guerra Civil ejerció como cirujano general al lado del Dr. D. Plácido González Duarte, con lo que adquirió desenvoltura y habilidad operatorias; a su término reanuda junto a su progenitor su especialización en el Hospital de La Princesa, a la par que mantiene una intensa y productiva actividad académica.

En 1944 el Dr. D. Enrique Pérez Castro le propone colaborar en la creación de una publicación urológica, en la que recoger la producción científica, dado el vacío existente en el país y, junto con Antonio Puigvert Gorro, fundan Archivos Españoles de Urología, en la que contribuyó con numerosas aportaciones (Figura 4). Acude a las sesiones científicas de la AEU y, en la celebrada en el mes de diciembre de 1945, expone un caso de Hemangioma renal. Se presenta, en 1946, a la oposición a la cátedra de Urología de la Faculta de Medicina de la Universidad Central, aprobó el primer ejercicio junto con los

TOMO VI

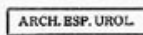

N UM. 3

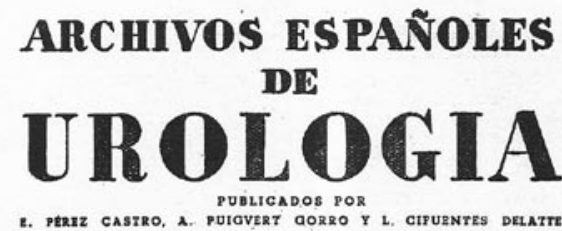

SUMARIO

originales

J. Sala de Pablo y L. Antón G. Pacheco: Algunas consideraciones

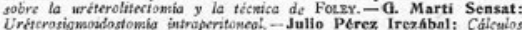
wertrales. - Jose M. Martinez Sagarra: Hipernefromats atipicos.-

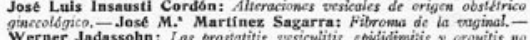

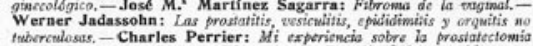

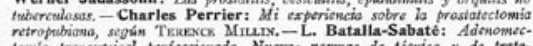

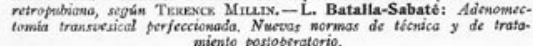
BIBLIOORAFIA

1950

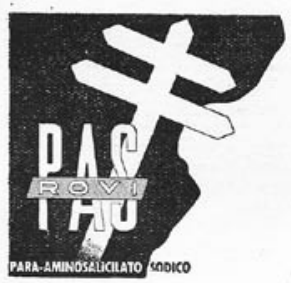

PROSIGUE LA LUCHA INCESANI CONTRA LA TUBERCULOSIS 3 FORMAS

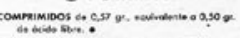

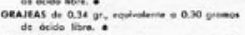

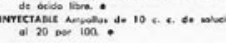
ROBIRT RIERA, S. A. IIA 
Drs. Alfonso de la Peña y Francisco Romero Aguirre, tras el segundo, la plaza le fue adjudicada al Dr. Peña. En 1947 aparece, en el Journal of Urology un artículo suyo sobre el epitelio trigonal y sus modificaciones; en el mes de julio fue nombrado miembro de número de la Sociedad Internacional de Urología en el transcurso del IX Congreso, celebrado en Suiza.

En 1950, con todo este extraordinario expediente, obtiene por concurso la plaza de jefe de servicio de Urología de la Beneficencia Provincial en el Hospital de la Princesa, de Madrid, a la jubilación de su padre, el Dr. Cifuentes Díaz (Figura 5), quien había ingresado por oposición como cirujano en el cuerpo de la Beneficencia en 1903; en 1908, al regreso de su residencia en París, en el Hospital Necker junto al Dr. Albarrán y en el Lariboisière con el Dr. Marión, fue encargado de proseguir con la consulta de Vías Urinarias, creada en el Hospital en 1890, en la que inició la asistencia el Dr. D. Manuel Barragán y Bonet (1861-1932), cirujano de valía dedicado a la atención de los pacientes urológicos; tuvo dos asistentes los Drs. Lluria Despau (1862-1925) y Suárez de Mendoza (n. 1849), formados en la escuela del Hospital Necker, de los que afirmó contribuyeron con mucho a ensanchar la especialidad, compartiendo los vastos conocimientos que habían aprendido del venerable maestro Dr. Guyón.

En 1910 fue ascendido a jefe de sala de Cirugía General, en la que simultaneaba estos enfermos con los de Urología, poco a poco consiguió que sus pacientes fueran en su mayoría de procesos urológicos; por su laboriosidad, por sus cuidados permanentes y por los excelentes resultados obtenidos logró que la dirección médica del centro lo aceptase como un hecho consumado en 1918 y, poco tiempo

\section{NOTICIAS}

EL DR. LUIS CIFUENTES DELATTE, JEFE DEL SERVICIO DE UROLOCIA DEL HOSPITAL DE LA PRINCESA, DE MADRID

En virtud de recientes oposiciones ha sido nombrado Jefe del Servicio de Urología del Hospital de la Princesa el Dr. Luis Cifurntes Delatte, que sucede en el cargo a su padre y maestro, el Dr. Pedro Cifuentes Díaz.

El día 29 de marzo pasado se celebró un banquete en el Hotel Ritz, organizado por un grupo de amigos, en honor del Dr. L. Cifuentes. Con el homenajeado se sentaron en la presidencia sus maestros los Dres. Pedro Cifuentes y Carlos Jiménez Díaz, D. Jacinto Benavente, D. Pedro Laín Entralgo, el Dr. González Duarte, el General Barroso, el Prof. X. Zubiri y los Dres. González Bueno, Lópzz Ibor, Sainz de Aja, García Orcoyen y D. José A. de Artigas. Entre los asistentes - cerca de trescientos comcnoales - se contaban las figuras más notebles de la Medicina madrileña, y en especial de la Urología.

Entre otras, fueron leídas adhesiones de D. Gregorio Marañón, D. Angel Pulide. D. Natalin Rivae D. Teńfiln Hernandn on tal Ninartar Camaral do Samidad

FIGURA 5. Noticia sobre la obtención de la plaza de jefe de servicio en el Hospital de la Princesa, aparecida en Archivos Españoles en 1950. después, se reconocía oficialmente por orden ministerial la creación del servicio de Urología. Desarrolló en él una gran carrera quirúrgica y docente, con la introducción de todas las técnicas de exploración y operatorias que el avance de la ciencia ofrecía, además de organizar cursos, el primero de ellos en abril de 1912, sobre "Cistoscopia, uretroscopia y cateterismo ureteral», a los que asistían numerosos médicos para perfeccionar conocimientos, con lo que lo convirtió en un referente urológico y un centro de formación para obtener el título de especialista al que acudieron además de su hijo Luis, entre otros, Jacinto Navas González (1898-1985), que realizó su actividad en Córdoba, Carlos Younger de la Peña (1920-1966), que ingresó de médico interno en el servicio de Urología en 1947 y, al siguiente año, lo hizo Ignacio Albalá Rodríguez, que ejerció en Alcoy desde 1952. Tuvo como colaboradores a los Drs. Hipólito Osuna Guerrero, Tomás Martín Luque, Guillermo Ontañón Carasa, Victoriano Molina García, Francisco Luque, José Ferrero Velasco y a Casas,

Con estos antecedentes se hizo cargo el joven Cifuentes del servicio de Urología con la responsabilidad no solo de mantener sino de elevar también el prestigio con que lo había dotado su padre y, a partir de ese momento, se entrega de pleno a la labor asistencial, sin desatender su capacidad intelectual como investigador y publicista.

En 1952 nos ofrece un resumen del trabajo efectuado en sus dos primeros años en el Hospital (Figura 6), lo que nos permite, a su vez, conocer el estado de la Urología en nuestro país durante esos años, en que la nefrectomía por tuberculosis, las operaciones por patología litiásica, las primeras cistectomías por carcinoma vesical, con la ureterosigmoidostomía como derivación, la consolidación de la resección transuretral de próstata y de los tumores vesicales, técnica de la que fue uno de los primeros en realizar, y la orquiectomía subalbugínea bilateral en los canceres de próstata, constituían la cirugía dominante.

En 1956 el vetusto edificio del Hospital es abandonado y trasladado a un nuevo centro ubicado en la confluencia entre las calles de Diego de León y Conde de Peñalver, inaugurado con el nombre de "Gran Hospital de la Beneficencia General del Estado", que mantendrá hasta 1984 en que, tras una remodelación, se le devuelve el primitivo de "Hospital de La Princesa". Disponía de sesenta camas en dos salas, una de hombres y otra de mujeres; creó allí un laboratorio de microbiología y dos cuartos para exploraciones cistoscópicas, ya que consideraba primordial el estudio inmediato con la visualización directa tanto de la orina como del interior vesical así como del tratamiento endoscópico de los tumores 
uroteliales por lo que preparó un quirófano para la cirugía transuretral en la misma planta; realizaba semanalmente sesiones clínicas que, dado su interés, eran muy frecuentadas por médicos de fuera del Hospital.

Se formaron numerosos urólogos a su lado, quienes distribuyeron la estela de su conocimiento por todo el país, de los que asistieron como alumnos libres en los años 1950-51 nos da sus nombres: José Luis Pérez Campoamor, que posteriormente ejerció en Oviedo, Cesáreo de la Fuente Llano, lo hizo en Toledo, Manuel Gil Noverques (1923-1982), que desarrolló toda su carrera en Valencia, José María Pons-Fuster (1923-2000), en Gandía (Valencia), José María Rico de Sanz, en Sevilla, Román Pereiro Minguez, en Vigo, Francisco Gómez Pérez, en Córdoba y V. Anlló Vázquez; no hemos podido identificar a Guillén, Muñoz, y Ortueta. Destacamos a Andrés Sánchez Salvador (1922-2006), colaborador asi-

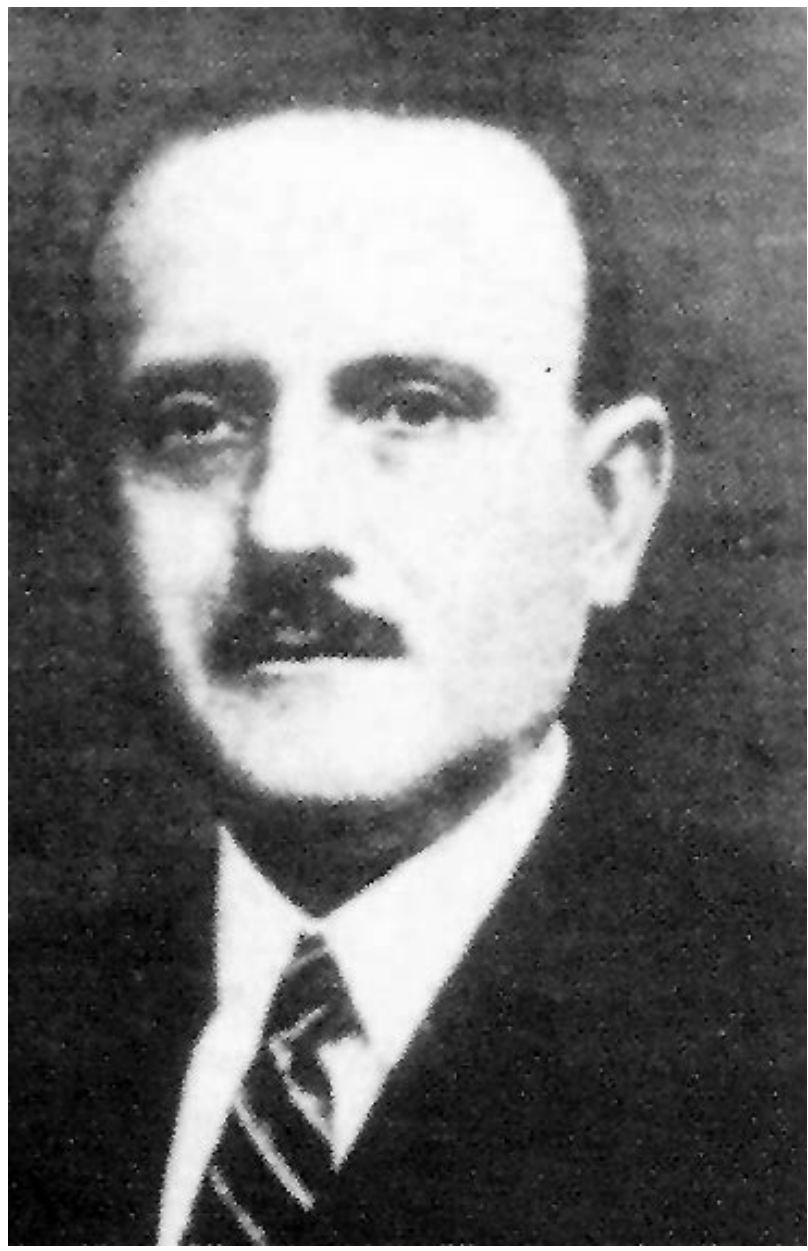

FIGURA 6. Dr. D. Pedro Cifuentes Díaz (1 880-1960). duo que desempeñó toda su carrera junto a él, en el puesto de jefe clínico y que fue quien lo sustituyó al frente del servicio de La Princesa en 1970. De los médicos que acudieron en cursos sucesivos señalamos, con ejercicio en Madrid, a Carlos Alférez Villalobos (1928-2007) que ocupó su cargo de jefe de servicio en la Clínica de Ntra. Sra. de la Concepción, a su jubilación en 1980, a Enrique Blanchard Castillo, que abandonó pronto la especialidad, a Benito Escudero Solano, que ingresó en Sanidad Naval, a Jesús Fraga Iribarne, a Mariano Rabadán Marina, a Antonio Palencia Anaya, fiel ayudante suyo personal y en el Hospital y a Ángel Escudero Barrilero, jefe del departamento de Urología en el Hospital Ramón y Cajal.

De provincias encontramos a Francisco Bañares Baudet, catedrático de Urología en la Universidad de La Laguna, en Tenerife, a Ernesto Canoa Galiana, en Vigo, al igual que José Luis Nogueira March, a Francisco Bellvert Ortiz, en Alicante, a Gerardo Server Falgás (1928-1999) en Murcia, junto con el que esto escribe, a Federico Rodríguez Rubio, en Cádiz, Cayetano del Valle Perate, en Las Palmas, a José Luis Perales Incas, en Algeciras, a Feliciano Alonso Sáiz y Jerónimo Fuente García, en Toledo, a Julián Nieto García, en El Ferrol, tras ejercer muchos años adscrito al Hospital en Diego de León, a Julio Zaragoza Orts, en Valencia, a Fernando Arocena Lanz, en San Sebastián, a José Manuel Mínguez Pemán, en Zaragoza, a Ignacio Pereira Sanz, actual responsable del servicio en La Princesa, y a Rafael Reyes Guijarro, en Cuenca, entre varios otros y además numerosos hispanoamericanos.

INFORME DEL SERVICIO DE UROLOGIA DEL HOSPITAL DE LA PRINCESA DE MADRID

(Años 1950 y 1951)

Dr. L. Cifuentes Delatte.

Fn la actualidad el Servicio de Urología del Hospital de la Princesa de Madrid cuenta con 44 camas, 22 de hombres y otras tantas de mujeres. Recientemente se han llevado a cabo las obras de reforma y ampliación que fueron aprobadas por la Dirección General de Beneficencia. Se ha construído un nuevo departamento de endoscopia, un despacho y secretaria de la Clínica y un Laboratorio urológico, con lo que han mejorado las facilidades de trabajo en el Servicio.

Las intervenciones quirùrgicas se realizan los mlércoles y sábados, el primero de estos đías đisponiendo de dos cuirófanos, lo que permite la actuación simultánee de dos equipos. de dos equipon to La consulta patca haciona,

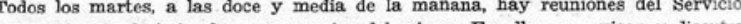
con asistencia de todos los componentes del mismo. En ellas se revisan y discutez luistorlas clinicas, radiografias, intervenciones quirúrgicas, etc., relativas a los en-

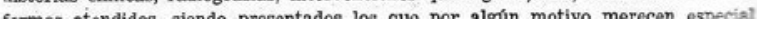

FIGURA 7. Informe sobre el servicio de Urología del Hospital de La Princesa en el curso 195 1-52. 
En 1970 pide la excedencia del Hospital de La Princesa y se dedica en exclusiva a la Fundación Jiménez Díaz, Clínica de la Concepción, de la que era jefe del servicio de Urología y donde cuenta con mayores medios y facilidad investigadora. Se forman en él, Remigio Vela Navarrete, actual responsable de la urología del centro, Armando Zuluoga, Enrique García Cuerpo, José Luis Rodríguez Miñón, Francisco Pastor Sempere, Jesús Salinas Casado, etc.

Publica en 1947 una de sus obras más trascendentales, Cistitis y Cistopatías (Figura 7), con la que inicia su estudio sobre los cambios histológicos que se producían en la mucosa vesical y que comenzó en Alemania con el profesor Hofman y, para lo que durante su estancia en Estados Unidos, recogió la bibliografía que necesitaba para ultimarla. Define

\section{LUIS CIFUENTES DELATTE}

\section{Cistitis}

y

\section{Cistopatías}

(176 miguras, de ELLAS 82 EN COLOR)

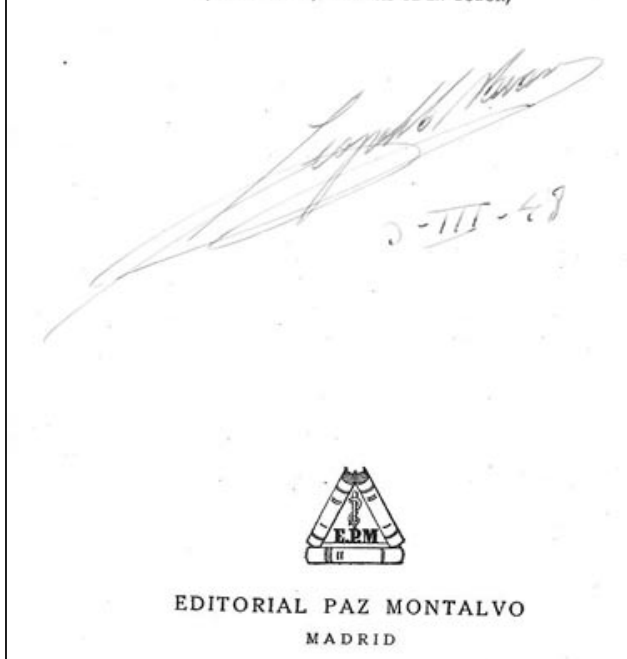

FIGURA 8. Cistitis y cistopatías, $1^{\underline{a}}$ edición, Madrid, 1947. la cistitis como uno de los capítulos más incompletos y peor conocidos de nuestra especialidad, en él aborda todas las causas inflamatorias y degenerativas que la producen y de las que, en el prólogo de su segunda edición, en 1961, afirma que no ha surgido en la bibliografía universal ninguna nueva obra que fuese dedicada concreta y especialmente a este problema lo que nos da una idea de la amplitud de su trabajo, de lo difícil del conocimiento de esta patología así como de la ardua labor de estudio que requirió su gestación y del esfuerzo de presentarla completamente renovada y reestructurada, trece años después, lo que le supuso revisar toda la bibliografía última aparecida sobre el tema.

En 1961 ve la luz el segundo de sus libros de mayor importancia, Cirugía Urológica Endoscópica (Figura 8), en la que vierte, fruto de su dilatada experiencia y amplio saber, la técnica de la resección transuretral de la próstata y de los tumores vesicales, expone con sencillez los pasos precisos para poder practicarla y llegar a dominarla y aclara muchos de los problemas que pueden surgir, por lo que ha constituido un el texto de consulta permanente para los urólogos, con él nos demuestra su clara vocación docente.

\title{
Cirugía urológica endoscópica
}

\author{
Fundamentos, indicaciones y técnica
}

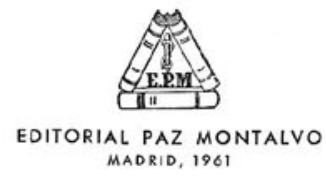

FIGURA 9. Cirugía endoscópica urológica: Fundamentos, indicaciones y técnica, $1^{\underline{a}}$ ed. Madrid, 1961. 


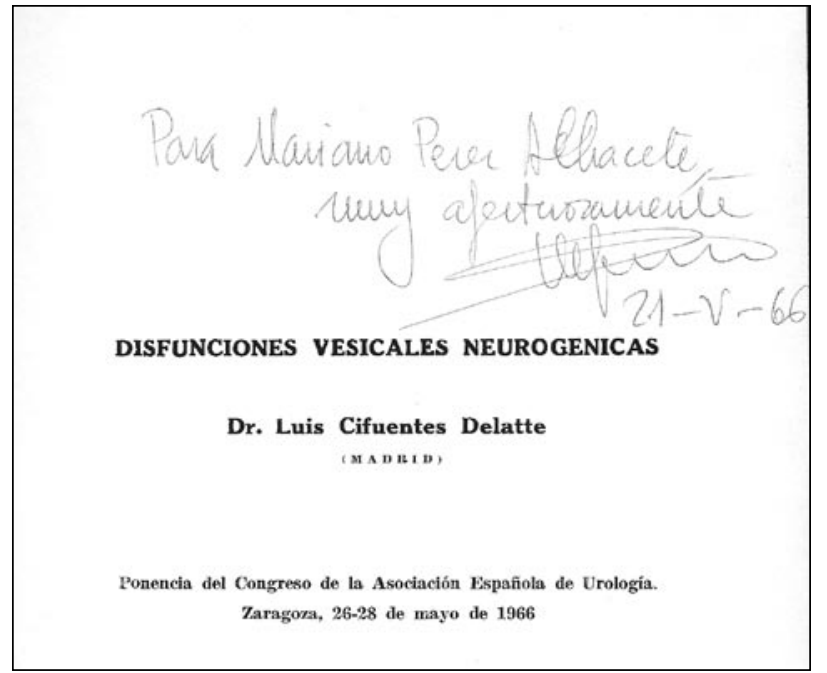

FIGURA 10. Disfunción vesical neurógena, ponencia del XXXI Congreso Nacional de Urología, Zaragoza, 1966

En la tercera, El laboratorio del urólogo, de 1974, centrada en el análisis del sedimento urinario en sus 156 páginas, nos ofrece una lección magistral en la que imbrica al especialista en la observación rutinaria de la orina en fresco, a pie del enfermo, como inestimable ayuda para un diagnóstico inme- diato, económico, sencillo de realizar y útil, y en el que señala paso a paso todos cuantos componentes se pueden encontrar en su sedimento, lo que permite avanzar en el diagnóstico.

Su cuarto tema de estudio lo protagoniza la litiasis urinaria, iniciado en los años 50 al poco de comenzar su labor en el Hospital de La Princesa, con el estudio de los cálculos de ácido úrico, lo que le condujo a lograr su disolución con un tratamiento alcalinizante acompañado de la ingestión de ácido cítrico; prosiguió con la investigación de la cristaluria oxolocálcica de la que un extracto de su trabajo fue publicado en la revista Science y con la del metabolismo fosfo-cálcico y del ácido oxálico, por medio de la espectrografía de infrarrojos y más adelante, de los cortes finos de cálculos observados con luz polarizada y, por último, el análisis de los núcleos litiásicos con microscopía electrónica de barrido.

Con el descubrimiento del corynebacterium urealítico, como agente productor de los cálculos infecciosos, culminan sus treinta años de trabajo en este campo que quedan recogidos en 1984, en su obra Composición y estructura de los cálculos renales.

La evolución de la vejiga neurógena es otro de los temas en los que centra su investigación para

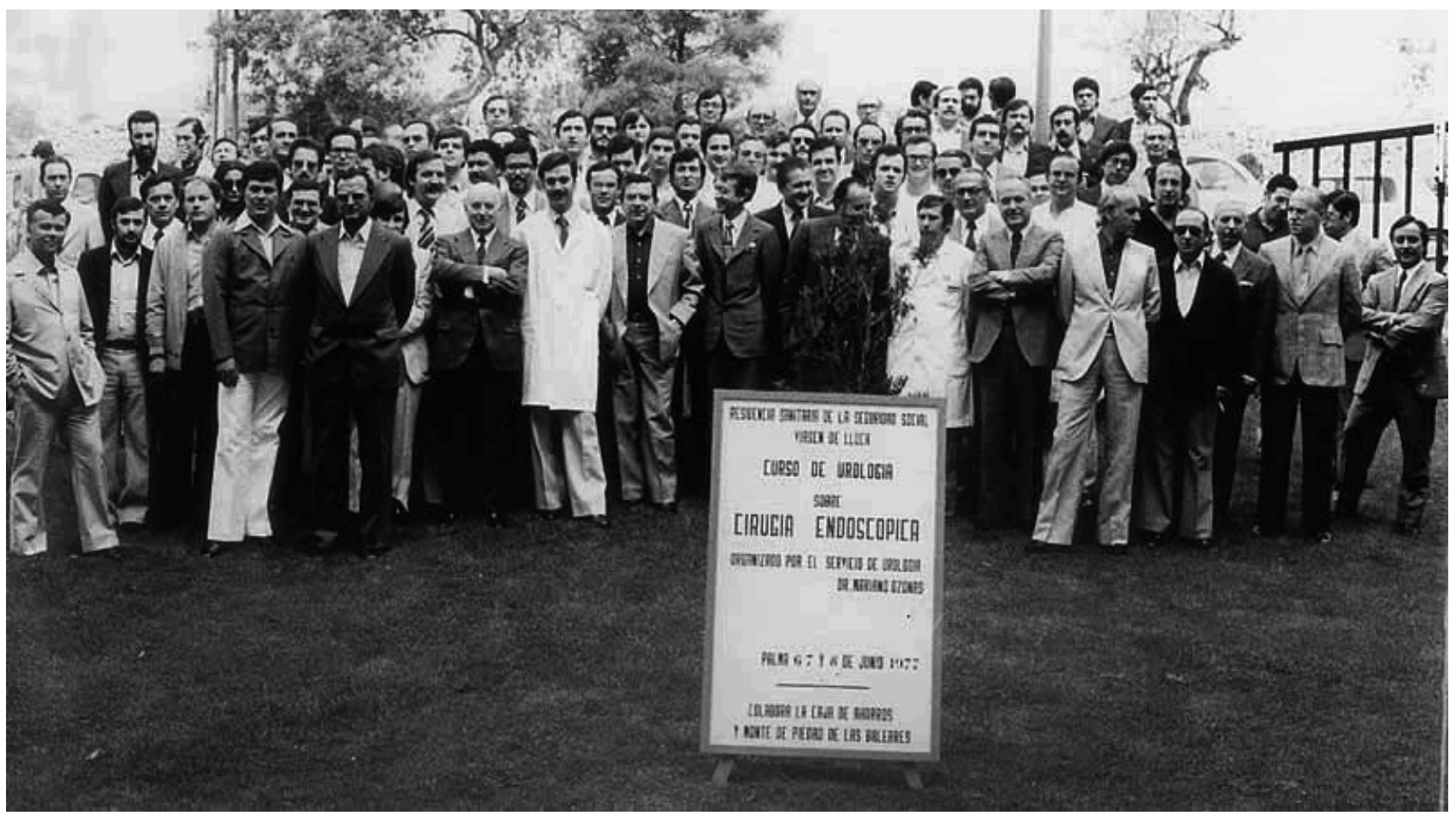

FIGURA 1 1. El Dr. Cifuentes en un curso sobre Endoscopia, realizado en Palma de Mallorca, en 1977. 
desentrañar sus mecanismos, brillantemente expuesto en la ponencia del XXXI Congreso Nacional de Urología, celebrado en Zaragoza, en 1966, Disfunciones vesicales neurógenas (Figura 9) en la que recoge los conocimientos anatomo y fisio-neurológicos vesicales que se tenían, y gran observador, los aplica en el tratamiento de su casuística de la que nos ofrece la respuesta clínica y terapéutica obtenida.

La patología tumoral vesical le atrae desde los inicios de la exploración endoscópica (Figura 10) y sus estudios sobre la citología urinaria y sobre cómo clasificar el tumor y saber su posible evolución son motivo de su preocupación, para ello realiza un análisis estadístico sobre 1.000 casos, que publica en el Journal francés de Urología, en 1971 y completa el seguimiento, que da a conocer en Archivos Españoles, cuatro años más tarde, junto con la recopilación de 70 compañeros con lo que logra 17.416 historias clínicas.

No queremos, por último, dejar de señalar el papel que ocupa la redacción de algunas de sus vivencias en las que nos muestra la Urología que le tocó vivir y le introduce, además, en la faceta de historiador de la que en su bibliografía encontramos varios ejemplos.

Fue profesor de Urología en la Universidad Autónoma de Madrid desde 1972, en el mismo año en el que ingresó en la Real Academia Nacional de Medicina con su discurso Origen y desarrollo de los cálculos renales. Socio de número de la Academia Médico Quirúrgica Matritense, fue secretario en la junta directiva de la AEU, desde 1952 hasta 1963, asociación que reconoció su mérito con la Medalla Francisco Díaz, en 1976, por su gran labor y entrega en pro de la Urología, como la más señalada de las otras muchas distinciones.

Todos estos capítulos fueron el leif motiv que lo estimuló a lo largo de su longeva vida. Persona amable y simpática, de trato agradable, fácil y ameno conversador, de amplia cultura, pero sobretodo maestro, querido y respetado, falleció en Madrid, el 15 de febrero de 2005.

\section{PUBLICACIONES}

- Obstrucción ureteral doble por intensa piuria. Bolaños y Aguilar, Madrid, 1934.

- Alteraciones del equilibrio ácido base en las nefropatías, tesis doctoral, ed. E. Berdejo, Zaragoza, 1935.
- Nefropatías (enfermedad de Bright), ed. Salvat, Madrid, 1943.

- Cistitis y cistopatías, ed. Paz Montalvo, Madrid, 1947.

- 2⿳a edic. edit. Paz Montalbo, Madrid, 1961.

- Reedición, ed. BOK, Madrid, 1989.

- Cirugía endoscópica urológica: Fundamentos, indicaciones y técnica, ed. Diana, Madrid, 1961.

- $2^{\underline{a}}$ edic. Paz Montalvo, Madrid, 1980.

- La litiasis renal y su relación con la dieta alimenticia. Ponencia a la V Conferencia Internacional de las Fuerzas Armadas. Diana Art. Gráf., Madrid, 1963.

- Disfunciones vesicales neurógenas, ponencia XXXI Congreso Nacional de Urología, Arch. Esp. Urol., núm. extra, Madrid, 1966.

- Origen y desarrollo de los cálculos renales, discurso de ingreso en la Real Academia Nacional de Medicina, 93 págs., Madrid, 1972.

- Tejido óseo en cálculos de aparato urinario (cinco observaciones). Graf. Orbe, Madrid, 1973.

- Historia de la prostatectomía. Un breve esquema evolutivo. Anal. Real Academia de Medicina. XCl: 3, 1974.

- El laboratorio del urólogo. Salvat edit., Barcelona, Madrid, Buenos Aires, 1974.

- Problemas actuales de Urología. Salvat edit., Barcelona, 1977.

- Composición y estructura de los cálculos renales, Salvat, edit., Barcelona, Madrid, Buenos Aires, 1984.

- Palabras sin imágenes. Comentarios a la evolución de la Urología, discurso de inauguración del curso 198687 en la Real Academia Nacional de Medicina, Madrid, 1986.

\section{Selección de artículos varios en publicacio- nes españolas:}

- Contribución al estudio de las cistitis, La Medicina íbera, 28 de octubre de 1932.

- Los focos extraurinarios en las cistitis. Actas IX Congreso Nacional de Urología. Cádiz, 1935. 
- El equilibrio ácido base en las nefropatías. Actas IX Congreso Nacional de Urología. Cádiz, 1935.

- Formaciones polipoideas del cuello vesical en la mujer. Arch. Esp. Urol. I (3): 234-245, 1944.

- Los nidos epiteliales de la mucosa del aparato urinario. Arch. Esp. Urol. III (1): 29-43, 1946.

- Las indicaciones y técnica de la ureterostomía. Arch. Esp. Urol. V (1): 57-66, 1948.

- La resección trasuretral en las obstrucciones del cuello vesical. Arch. Esp. Urol. VI (2): 3-41, 1949.

- Fístulas recto-uretrales congénitas. Arch. Esp. Urol. VII (2): 101-114, 1951.

- Disfunción vesical neurógena tratada con resección de los nervios erectores. Arch. Esp. Urol. VIII (1): 31 . 44, 1952.

- Regresión y dilataciones de pelvis y uréter después de implantaciones urétero-intestinales (implantación directa en valvas). Arch. Esp. Urol. IX (3): 179-196, 1953.

- Cristaluria, litiasis úrica y gota. Rev. Clín. Esp. 59: 139-145, 1955.

- La eliminación renal de ácido úrico en los gotosos con cristaluria o litiasis úrica. Arch. Esp. Urol. XI (3): 165-185, 1955.

- Técnica de la resección endoscópica de los papilomas vesicales. Arch. Esp. Urol. XII (4): 215-230, 1956.

- Homenaje al profesor J. Albarrán. Arch. Esp. Urol. XV (3): 206-265, 1962.

- Bases fisiopatológicas para el tratamiento de la litiasis renal. Ann. Real Acad. Med.-Quir. 49, 1.035, 1965.

- Calcio, fósforo y cocientes $\mathrm{Ca} / \mathrm{P}$ en los sedimentos fosfáticos. Arch. Esp. Urol. XVIII (4): 263-270, 1965.

- Tejido óseo en cálculos del aparato urinario. Arch. Esp. Urol. XXVI (5): 457-480, 1965

- Los fosfatos cálcicos de la serie apatítica. Rev. Clín. Esp. Año XXVI (6): 345-357, 1965.

- Resultado de análisis químico cualitativo de 400 cálculos del aparato urinario. Arch. Esp. Urol. 22 (1):
35-55, 1969.

- Tratamiento de la Hipercalciuria idiopática con celulosa fosfato sódica, Rev. Clín. Esp. Año XXX, 119 (1): 61-66, 1970

- La gammagrafía ósea con estroncio 85 en el diagnóstico de las metástasis óseas del cáncer de próstata. Arch. Esp. Urol. 25 (4): 383-409, 1972.

- Litiasis renal úrica y sus relaciones con la gota. Bol. Fun. Jiménez Díaz, 4: 13-22, 1972.

- Frecuencia de las neoplasias vesicales en ambos sexos. Una encuesta entre los urólogos españoles. Arch. Esp. Urol. 28 (4): 335-351, 1975.

- Eficacia del tratamiento local con Tiothepa en las neoplasias vellosas múltiples y recidivantes de la vejiga. Arch. Esp. Urol. 29 (6): 629-639, 1976

- Carcinoma in situ de vejiga. Presentación de seis casos. Arch. Esp. Urol. 30 (6): 537-561, 1977.

- Enseñanza de la cirugía endoscópica. Actas Urol. Esp. III (6): 303-306, 1979.

- Enrique Pérez Castro (1908-1980) (necrológica). Arch. Esp. Urol. 33 (6): 523, 1980.

- Ultra estructura del carcinoma urotelial. Actas Urol. Esp. 34 (4): 199-206, 1980.

- Historia del estudio microscópico de los cálculos del aparato urinario en el siglo XIX. Arch. Esp. Urol. 34 (5): 375-384, 1981.

- Cálculo de sulfato cálcico de origen renal. ¿Una observación homologable? Arch. Esp. Urol. 36 (3): 117-124, 1983.

- 40 Aniversario de Archivos Españoles de Urología. Arch. Esp. Urol. 37 (1): 1-5 1984.

- Infecciones urinarias por diferentes ureolíticos con cultivos falsamente negativos. Arch. Esp. Urol. 37 (4): 329-337, 1984.

- Mi primer Congreso. Actas Urol. Esp. núm. extra: 9-12, 1985.

- Núcleo con tubos renales calcificados en el interior de un pequeño cálculo de whewelita. Arch. Esp. Urol. 39 (1): 71-75, 1986.

- Presencia de ácido oxolínico en un cálculo renal. Arch. Esp. Urol. 39 (5): 375-379, 1986. 
- El tratamiento alcalinizante como preventivo de la litiasis oxolo-cálcica recidivante. Arch. Esp. Urol. 44 (8): 903-913, 1991.

- Mi aprendizaje de la cirugía endoscópica (EE.UU., 1926). Arch. Esp. Urol. 46 (3): 167-180, 1993.

- El 50 aniversario. Arch. Esp. Urol. 47 (6): 548 $553,1994$.

- La clínica urológica de Von Lichtemberg en Berlín (1933). Arch. Esp. Urol. 48 (2): 105-1 12, 1995.

\section{BIBLIOGRAFÍA y LECTURAS RECOMENDADAS ( ${ }^{*}$ lectura de interés $y^{* *}$ lectura fundamental)}

*1. «El Dr. Cifuentes Delatte, jefe del servicio de Urología del Hospital de la Princesa, de Madrid», Arch. Esp. Urol, VI (2): 206, Madrid, 1950.

2. CLEMENTE RAMOS, L., Historia Biográfica y Bibliográfica de la Urología Española en el siglo XX, pág. 278, Edicomplet, Madrid, 2002.

*3. ESCUDERO BARRILERO, Á., «D. Luis Cifuentes Delatte, D. Luis: Clínico, cirujano e investigador». Actas Urol. Esp., monográfico: 10-20, 2006.
**4. INSAUSTI CORDÓN, J. L., «Compendio histórico de la Urología española y de su Asociación», ponencia IV Congreso Hispano Americano de Urología, pág. 107, Acapulco Reycosa, Madrid, 1982.

5. GARCÍA CUERPO, E., Historia Biográfica y Bibliográfica de la Urología Española en el siglo XX, pág. 251, Edicomplet, Madrid, 2002.

6. *OLIVA, H.; MANZARBEITIA, F.; VELA NAVARRETE, R., «Histología y anatomía patológica en la obra de Luis Cifuentes: de las cistopatías al carcinoma intraepitelial». Actas Urol. Esp., monográfico: 6-10, 184-85, 2006.

7. PEREIRA SANZ, I., «Hospital de la Princesa y D. Luis Cifuentes». Actas Urol. Esp., monográfico: 67-70, 2006.

8. PÉREZ ALBACETE, M., «La docencia urológica en España la primera mitad del siglo XX».Urol. Integr. Invest. 7 (2): 191-198, 2002.

9. IBID., 100 Figuras de la Urología Española. Tip. San Francisco, Murcia, 2005.

*10. RODRÍGUEZ RUBIO, F., «Luis Cifuentes y la resección transuretral de próstata». Actas Urol. Esp., monográfico: 59-68, 2006.

*11. VELA NAVARRETE, R., «Luis Cifuentes: de cistitis y cistopatías (1947) a composición y estructura de los cálculos renales (1984)», Urol. Integr. Invest., 7 (2): 187-190, 2002.

*12. VELA NAVARRETE, R., «Luis Cifuentes y su escuela. Datos biográficos de un extraordinario hombre de ciencia, urólogo y académico», Actas Urol. Esp., monográfico. 1-5, 2006. 